\title{
Abstracts der 29. Jahrestagung der Gesellschaft für Pädiatrische Gastroenterologie und Ernährung e.V. (GPGE)
}

\author{
Dortmund, 26.-29. März 2014
}

U nsere diesjährige GPGE-Jahrestagung findet mit Dortmund an einem Traditionsort der Kindergastroenterologie statt. Schon 1975, also vor fast 40 Jahren, hat sich die „Pädiatrische Arbeitsgruppe, Gastroenterologie und Ernährung" zu ihrer 2. wissenschaftlichen Tagung hier getroffen.

Die Stadt und ihre Region, aber auch unser Fachgebiet haben sich seither weiterentwickelt und in manchen Aspekten grundlegend verändert. Diesem Wandel verleihen das Tagungsgebäude, der "Congress on Tour" als auch ganz besonders das wissenschaftliche Programm Ausdruck.

Als moderne Querschnittsthemen haben wir die Immunologie, die Psychosomatik sowie Betreuungs- und Versorgungsaspekte ausgewählt. Diese Themenbereiche haben eine wachsende Bedeutung für die Kindergastroenterologie, sowohl in praktischer als auch in wissenschaftlicher Hinsicht. Weitere Hauptthemen werden seltene Erkrankungen des Ösophagus und Kolon sein, also zwei klassische kindergastroenterologische Themenkomplexe.

Die Jahrestagung bietet darüber hinaus die Gelegenheit, wissenschaftliche Beiträge und Kasuistiken aus dem vielfältigen Fachgebiet der pädiatrischen Gastroenterologie, Hepatologie und Ernährung in Form von Postern und Vorträgen zu präsentieren und zu diskutieren. Die Beiträge wurden in einem unabhängigen Begutachterverfahren ausgewählt und werden auf den folgenden Seiten in Form von Abstracts publiziert.

Wir hoffen, dass die Auswahl der Beiträge Ihr Interesse weckt und freuen uns mit Ihnen auf den kommenden Kongress.

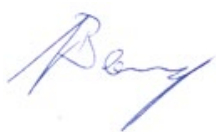

Dr. Jens Berrang

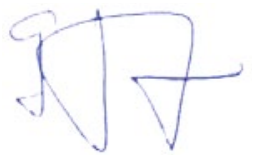

Guido Bürk

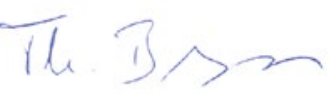

Dr. Thomas Berger
Tagungsdaten auf einen Blick

\section{Veranstalter}

Gesellschaft für Pädiatrische Gastroenterologie und Ernährung Vorsitzender: Dr. Stephan Buderus

Geschäftsstelle der GPGE:

Chausseestraße 128/129, 10115 Berlin

Tel.: 030/27582345, Fax: 03222/2455839

E-Mail: info@gpge.de, Internet:www.gpge.de

\section{Veranstaltungsort}

Dortmunder U

Zentrum für Kunst und Kreativität

Leonie-Reygers-Terrasse, 44137 Dortmund

Tagungsleitung

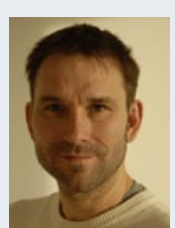

Dr. Jens Berrang

Klinik für Kinder- und Jugendmedizin

Klinikum Dortmund gGmbH

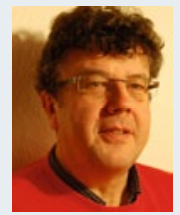

Guido Bürk

Paedicum Ruhrkidz

Herne

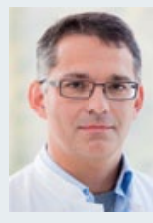

Dr. Thomas Berger

Vestische Kinder-Jugendklinik

Universität Witten/Herdecke

Organisation

Schmidt-Römhild Kongressgesellschaft $\mathrm{mbH}$

E-Mail: kongresse@schmidt-roemhild.com

Internet

www.gpge2014.eu 